\title{
Hufelands Opiumtherapie im zeitgenössischen Vergleich
}

Von Luzius von Rechenberg und Huldrych M. Koelbing

\section{Einleitung}

Für Christoph Wilhelm Hufeland (1762-1836) war das Opium Zeit seines Lebens ein Hauptpfeiler jeder ärztlichen Therapie. Zusammen mit dem Aderlaß und den Brechmitteln rechnete er es zu den drei Heroen, die dem Arzt zu Diensten standen und von deren richtigem oder verfehltem Gebrauch sein Erfolg oder Mißerfolg, sein guter oder schlechter Ruf abhingen (s. unten S.100). Diese Botschaft gab er noch im letzten Jahr seines Lebens seinen Kollegen als Vermächtnis seiner fünzigjährigen Erfahrung mit. Das Buch, das diesen Lobpreis des Opiums enthält und ausführlich begründet Hufelands «Enchiridion medicum» ${ }^{1}$ - fand, wie seine vielen Auflagen und Übersetzungen beweisen, eine große Zahl aufmerksamer Leser unter den Ärzten seiner Zeit und hatte damit einen beträchtlichen Einfluß auf die ärztliche Praxis. Es schien uns deshalb reizvoll, Hufelands Opiumtherapie kritisch zu analysieren und darzustellen. Dabei stellte sich die Frage, ob denn die Ausführungen des großen alten Mannes der damaligen deutschen Medizin noch auf der Höhe der Wissenschaft seiner Zeit standen und ihren neueren Ergebnissen Rechnung trugen. Schon gut 30 Jahre zuvor, 1805, hatte ja Friedrich Wilhelm Adam Sertürner (1783-1841) aus dem Opium das Morphin isoliert ${ }^{2}$, und seit 15 Jahren gab es François Magendies «Formulaire pour la préparation et l'emploi de plusieurs nouveaux médicamens, tels que la noix vomique, la morphine [etc.]» (Paris 1821), den ersten bedeutenden Versuch, die Pharmakotherapie auf streng naturwissenschaftlicher Grundlage exakt und experimentell zu entwickeln. Es stand zu befürchten, daß Hufeland diese neue Entwicklung, die in Frankreich einsetzte, ebensowenig mitvollzogen hatte, wie er den Wert des Stethoskopes, der Laennecschen Auskultation des Herzens und der Lungen für die diagnostische Praxis erkannte $^{3}$. Die Befürchtung wurde durch den Vergleich mit führenden medizinischen Nachschlagewerken aus Frankreich und Deutschland bestätigt. Das Ergebnis dieser Untersuchung - sie bildet den Inhalt der Doktorarbeit des Erstautors dieser Arbeit ${ }^{4}$ - soll im folgenden in gedrängter Form mitgeteilt werden. 


\section{Die Quellen}

Hufelands «Enchiridion medicum» (vgl. Anm.1) war nach seinem Erscheinen 1836 sofort vergriffen, so daß der Autor im selben Jahr noch eine «zweite vermehrte Auflage» verfaßte. Die folgenden Auflagen erschienen postum.

Die Bedeutung des «Enchiridion medicum» ergibt sich aus der Zahl seiner Auflagen - die zehnte und letzte kam 1857 in Berlin heraus - und der Übersetzungen. 1838 wurde in Amsterdam eine holländische Fassung publiziert, im selben Jahr eine französische in Brüssel, drei Jahre später durch einen anderen Übersetzer wiederum eine französische in Brüssel. Die englische Übersetzung, die in New York herausgegeben wurde, erlebte zwei Auflagen, 1842 und 1844. Eine italienische Version erschien 1851 in Bologna. Eine russische Übersetzung ist undatiert. 1869 erschien in Žitomir noch eine hebräische Fassung ${ }^{5}$.

Durch all diese Ausgaben des «Enchiridion medicum» beeinflußte Hufeland die praktischen Ärzte weit über seinen Tod hinaus. Julius Petersen bezeichnet das «Enchiridion» als «Norm für den Praktiker» ${ }^{6}$. Ein Beispiel für einen solchen Praktiker ist sicher Abraham Maret (1783-1866), ein Emmentaler Arzt und Freund von Pfarrer Albert Bitzius - Jeremias Gotthelf. Er soll den stereotypen Ausdruck verwendet haben: «Hufeland sagt ...» Die Arztfiguren im Werk von Jeremias Gotthelf und seine Krankheitsbeschreibungen sind nach Carl Müller durch die Vermittlung Marets nachhaltig von Hufeland beeinflußt ${ }^{7}$.

Der Schweizer Arzt und Gesundheitspolitiker Jacob Laurenz Sonderegger (1825-1896) schreibt dagegen im Rückblick auf seine praktisch-ärztliche Tätigkeit: «Ich gehörte zu der ersten Generation, der es vergönnt war, die anatomische und physiologische Auffassung am Krankenbette zu verwerten und die physikalische Diagnostik zu handhaben, wobei man allerdings weiter kam, als bei der hergebrachten Hufeland'schen Praxis» ${ }^{8}$. Hufeland war also immer noch ein Bezugspunkt.

Zum Vergleich mit Hufeland und als Standard der damaligen wissenschaftlichen Medizin dienten uns die folgenden vier Nachschlagewerke französischer und deutscher Sprache:

"Dictionaire des sciences medicales», Bd.37, Paris 1819, herausgegeben von Charles-Louis-Fleury Panckoucke (1780-1844) in 60 Bänden, Paris 1812-1822. François-Victor Mérat de Vaumartoise (1780-1851) redi- 
gierte den «Dictionaire» vom elften Band an. Etwa 250 Artikel, so auch derjenige über das Opium, stammen von ihm selbst.

«Encyclopädie der medicinischen Wissenschaften», Bd.9, Leipzig 1832, eine Übersetzung und freie Bearbeitung der ersten Ausgabe des «Dictionnaire de médecine» (21 Bände, Paris 1821-1828) durch Friedrich Ludwig Meissner (1796-1860), die in 13 Bänden 1830-1834 in Leipzig erschien. Karl Christian Schmidt (1792-1855) trat vom sechsten Band an als Mitherausgeber an die Seite Meissners. Schmidt war von 1834-1843 Redakteur der bekannten «Jahrbücher der in- und ausländischen gesammten Medicin». Der erste Teil des Artikels über das Opium, der sich vor allem mit seiner Zusammensetzung befaßt, ist mit dem Namen von Achille Richard (1794-1852) signiert. Richard war Professor der Naturgeschichte an der medizinischen Fakultät von Paris und schrieb vor allem über medizinisch-botanische Themen ${ }^{9}$. Der zweite - wesentlich längere und wichtigere - therapeutische Teil des Artikels stammt von Louis Benoît Guersant (auch Guersent, 1777-1848), Direktor des «Hôpital des enfants malades» in Paris und Agrégé für das Fach Therapie an der medizinischen Fakultät. Offenbar handelt es sich beim Opium-Artikel der «Encyclopädie» um eine reine Übersetzung durch Meissner und/oder Schmidt.

«Dictionnaire de médecine», Zweite Auflage, Bd.22, Paris 1840. Für die zweite Auflage des «Dictionnaire de médecine», die in Paris von 1832-1846 in dreißig Bänden erschien, schrieb Guersant wiederum den therapeutischen Teil des Opium-Artikels. Gegenüber der ersten Ausgabe ist er viel länger geworden. Der Apotheker Eugène Soubeiran (1793-1858) verfaßte die Abschnitte über Geschichte, Chemie und Pharmakologie des Opiums. Von Jean-Gaston-Marie Blache (17991871), Guersants Schwiegersohn, stammt der Abschnitt über die Opiumvergiftung, von Mathéo José Bonaventura Orfila (1787-1853), dem Begründer der forensischen Toxikologie, derjenige über die gerichtsmedizinischen Untersuchungen bei einer solchen.

"Universal-Lexicon der practischen Medizin und Chirurgie», Bd.10, Leipzig 1841. Die Grundlage dieses Lexikons ist der «Dictionnaire de Médecine et de Chirurgie pratiques», der in 15 Bänden 1829-1836 in Paris erschien und bekannte Männer wie Andral, Bégin, Bouillaud, Magendie, Rayer u. a.m. als Autoren nannte. Die 2. Auflage kam 1837 in Brüssel heraus. Die deutsche Fassung ist «frei bearbeitet so wie mit den allgemeinen und besonderen Grundsätzen und practischen Erfahrungen aus dem Gebiete 
der Homöopathie bereichert von einem Vereine deutscher Ärzte». Die 14 Bände wurden 1835-1848 in Leipzig publiziert. Herausgeber war bis zum 11. Band (1843) ein Dr. Pestel, danach der Leipziger Arzt Dr. Carl d'Alnoncourt (geb. 1800). Er hatte beabsichtigt, das Universal-Lexicon durch Supplemente weiterzuführen, mußte aber, nach dem Scheitern der deutschen Märzrevolution, im Sommer 1848 Deutschland verlassen. ${ }^{10}$ Die einzelnen Artikel des Universal-Lexicons enthalten zahlreiche redaktionelle Einschübe und werden dadurch insgesamt zugleich anonymer wie enzyklopädischer. Der therapeutische Teil des Artikels «Opium» stammt ursprünglich von Fernand Martin-Solon (1795-1856), Arzt am Hôpital Beaujon in Paris; die Redaktion zieht jedoch u. a. ausgiebig eine Arbeit des Arztes Karl Georg Neumann (1774-1850) zur Ergänzung heran, der 1819-1828 dirigierender Arzt und Dozent an der Berliner Charité gewesen war.

\section{Die Opiumtherapie in Hufelands «Echiridion medicum» (1836)}

\subsection{Hufelands Wertung der Opiumtherapie}

Hufeland zählt das Opium, wie eingangs erwähnt, zu den drei wichtigsten Heilmitteln: «Es gibt drei Mittel in der Heilkunst, welche als Magnaten und Anführer des übrigen Streitheers hervorragen. Sie sind: das Aderlaß, das Brechmittel, und das Opium. Sie repräsentieren gleichsam die drei Fundamentalmethoden der Heilkunst, die antiphlogistische, die excitierende; und zugleich die drei Grundsysteme des Organismus, das Aderlaß das irritable, das Brechmittel das reproduktive, und das Opium das sensible. Sie greifen unmittelbar ins Leben selbst ein, und sind die drei entscheidendsten und schnellwirkendsten Mittel in dem ganzen Arzneivorrath, - die wahren $\mathrm{He}$ roica; - sie können das Leben und auch den Tod geben; sie entscheiden den Kampf in dem kritischen Augenblick; von ihrem richtigen Gebrauch hängt das Glück und der Ruf des Arztes vorzüglich ab. Sie sind ganz einzig, jedes für sich, und durch nichts zu ersetzen. Wer diese drei recht anzuwenden wei $\beta$, der ist der Meister, und daran ist er zu erkennen» (S.805). ${ }^{11}$

Im ersten Hauptteil des «Enchiridion medicum», der nach Krankheitsbildern gegliedert ist, wird das Opium unzählige Male erwähnt. In dem kurzen zweiten Teil, der speziell den «drei Kardinalmitteln der Heilkunst» gewidmet ist, beschreibt Hufeland die Wirkungsweise und den Gebrauch des 
Opiums ausführlich. Ein Zitat aus der «Opiologie» von Wolfgang Wedel (1674, 2. Aufl. 1682), welches Hufeland als Einleitung zum Abschnitt über das Opium benützt, beleuchtet seine zwiespältige Haltung gegenüber diesem Medikament: «Sacra vitae anchora, circumspecte agentibus, est Opium, Cymba vero Charontis in manu imperiti - Es ist ein zweischneidiges Schwerdt, eine göttliche Himmelsgabe in der Hand des Meisters, das tödtlichste Gift in der Hand des Unkundigen.» Im ganzen «Enchiridion medicum» preist Hufeland die Opiumtherapie, warnt aber auch immer vor deren nachteiligen Folgen.

Obwohl für Hufeland die Opiumtherapie so wichtig ist, schätzt er die chemische Analyse des Opiums gering. Seine Begründung dafür erklärt sich durch seine vitalistische Grundhaltung. «Unstreitig bleibt das erste und wichtigste: die Erkenntnis und Bestimmung der Grundwirkung, des wesentlichen Charakters, eines Mittels. [...] Eben so wenig reicht die chemische Analyse, und sei sie auch noch so genau, dazu hin, die ja noch immer der Autonomie und Autokratie des Lebens untergeordnet bleibt» (S.820).

Immerhin erwähnt er kurz die einzelnen Bestandteile des Opiums, wenn auch nur unvollständig, und hebt das Morphium als wichtigste Komponente hervor. Danach verurteilt er aber erneut die chemische Analyse und sagt: «[...] die Wirkung des Opiums geht hervor aus der Vereinigung all dieser Stoffe, $[\ldots]$ aus der ganz eigenthümlichen Art der Verbindung und des Daseins, die aber die chemische Analyse zerstört.» Für den ersten Teil des Zitates beruft sich Hufeland auf Orfila, den zweiten setzte er selbst hinzu. Dies widerspricht der Auffassung Orfilas, der im «Dictionnaire de médecine» im Abschnitt «Recherches médico-légales sur l'empoisonnement par l'opium et ses composés» schreibt: ${ }^{12}$ «... nous croyons pouvoir établir, que l'opium doit ses propriétés vénéneuses a un sel de morphine, au principe de Derosne [Narkotin], à la codéine, et probablement à une autre matière qui n'a pas encore été séparée, [...] que l'action de l'opium paraît résulter de l'action combinée de ces matières...»

Die Ablehnung der chemischen Analyse des Opiums durch Hufeland hat weitreichende Folgen. Er empfiehlt den Gebrauch des Gemisches Opium anstatt des reinen Morphinsalzes (S.830). Er hat offensichtlich keine Kenntnis von den vielen Experimenten mit den Bestandteilen des Opiums, die vor allem in Frankreich die Grundlagen der Opium- resp. der Morphintherapie wesentlich erneuert haben, und seine Dosierungsangaben sind sehr ungenau verglichen mit den klaren Richtlinien, die zu dieser Zeit für das Morphinsalz bereits bestanden (vgl. Abschnitt 4.3.) 


\subsection{Wirkung und Wirkungsweise des Opiums}

Hufeland spricht zuerst von den «konstanten Phänomenen» (S.821) und schließt daraus auf eine «Grundwirkung» (S.824ff.) des Opiums. Zum Schluß erklärt er die «Wirkungsart» (S.826ff.) des Opiums, wobei er eigene und fremde Gedanken aufführt. Er nennt acht konstante Phänomene (S. $821 \mathrm{ff}$.$) :$

1. «Der Puls wird gehoben, voll, kräftig.» Dieser Befund ist für Hufeland immer vorhanden, die Wirkung auf die Pulsfrequenz jedoch abhängig vom Zustand des Patienten und von der Dosis.

2. «Turgor (Expansion) des Bluts.» Hufeland spricht von «Blutkongestionen», die «zunächst nach dem Kopf, den Lungen, oder nach irgend einem anderen dazu disponierten Organ gehen.» Er verwendet auch den Begriff «Lebensturgor des Opiums, der sich in der Blutausdehnung zeigt», warnt aber zugleich vor der «Gefährlichkeit der Blutkongestion nach dem Kopfe.»

3. «Vermehrung der Lebenswärme», für Hufeland die direkte Folge des gesteigerten «Lebensturgors» und der «vermehrten Circulation.»

4. «Das Nervensystem, besonders das Sensorium, wird unmittelbar und mächtig afficirt, und zwar depotenzirend, Sensibilität vermindernd, denn es entsteht Betäubung, Schläfrigkeit, tiefer Schlaf, bei örtlicher Anwendung Betäubung, Unempfindlichkeit des Theils, Aufhören von Schmerzen und Krämpfen desselben.» Hufeland beschreibt auch, daß sich zuweilen auch «erhöhte Munterkeit, Exaltation des Sensoriums und des psychischen Leidens, ja Delirium bis zur Raserei» einstellt. Diese Wirkungen sind für ihn aber «höchst relativ, die depotenzirende Grundwirkung erhält [immer] die Oberhand.»

5. «Verstopfung des Stuhlganges und Trockenheit des Halses.» Für Hufeland wird durch die «innere Applikation» des Opiums eine «örtliche Lähmung des Darmkanals und seiner absondernden Gefäße» bewirkt.

6. «Vermehrte Hautabsonderung, Schweiß.» Hufeland erklärt dies als ein «Produkt der excitirenden Kraft, der vermehrten arteriellen Thätigkeit» und «der sedativen, der Lösung des Hautkrampfes, der Erschlaffung der Gefäßmündungen.»

7. «Wirkung auf die Geschlechtstheile und Urinwerkzeuge.» «Verliebte Träume, Erektionen, Ejakulationen» sind für Hufeland ziemlich konstante Wirkungen. Es ist hingegen nicht sicher, ob wirklich eine «Ver- 
mehrung der Harnabsonderung, oder nur eine Vermehrung des Reizes zur Harnausleerung» zu beobachten ist.

8. «Bei zu starkem oder anhaltendem Gebrauch: Auflösung des Bluts, Zersetzung der organisch-vitalen Bindung und Mischung, Putrescenz, Gangrän, schneller Übergang in völlige Fäulnis nach dem Tode.» Hufeland betont warnend, daß «alle hitzigen Fieber durch zu starken Gebrauch des Opiums in Faulfieber, alle Entzündungen in Brand verwandelt werden können.»

Im anschließenden Kapitel «Grundwirkung» (S. 824 ff.) versucht Hufeland, die eben besprochenen «Phänomene» auf zwei grundlegende Effekte des Opiums zurückzuführen: «Herabstimmung, ja gänzliche Aufhebung der Sensibilität, des Nervenlebens, und Hervorrufung und höhere Potenzierung der Irritabilität des Herzens und des ganzen Blutlebens.» Er nennt das Opium «das größte Exitans und Cardiacum - im eigentlichen Wortverstand ein herzstärkendes Mittel».

Nach einem historischen Überblick über die verschiedenen bereits bestehenden Erklärungen der Wirkung des Opiums entwickelt Hufeland im Kapitel «Wirkungsart» (S.826ff.) seine eigenen Ideen zur Entstehung der genannten Phänomene.

Hufeland vergleicht zunächst den Geruch des Opiums mit jenem anderer narkotischer Pflanzen wie Hyoscyamus und Stramonium und schließt daraus auf die Existenz eines «narkotischen Stoffs». Dieser narkotische Stoff wirkt «unmittelbar und specifisch auf das Gehirn und Nervensystem». Er beweist dies durch die örtliche Anwendung von Opium, die «schmerzstillend und besänftigend» wirkt, oder «schlafanstoßend» beim Opiumpflaster auf den Schläfen. Er betrachtet den Dunst des Opiums als die reinste Darstellung des «narkotischen Stoffs», wobei dieser flüchtige Dunst auch in der «Aqua opiata», dem wäßrigen Extrakt des Opiums, enthalten ist. Hufeland beruft sich auf seine Erfahrung, wenn er sagt, daß bei der örtlichen Anwendung des Opiums, der Anwendung des Dunstes oder des wäßrigen Extraktes das Blutsystem nicht aufgeregt werde. All dies betrachtet er als Beweis für «das Dasein eines narkotischen Stoffs, der ohne Vermittlung der Blut- und Gefäßaufregung wirksam ist». In der damaligen vitalistischen Ausdrucksweise wurde die vermeintliche Vermittlung der Opiumwirkung durch die sensiblen Nerven mit dem Begriff der Sympathie erklärt.

Hufeland schließt das Kapitel «Wirkungsart» mit dem Satz, daß das Opium «unmittelbar auf die Vitalität selbst und auf alle Punkte, Modifika- 
tionen und Äußerungen derselben wirkt, sie durchdringt und erfüllt, $[\ldots]$ daß es die organisch-vegetative Sphäre des Lebens, den fundamentalen plastischen Lebensproceß erhöht, die sensible Sphäre hingegen deprimirt».

Es wird dem heutigen Leser des Kapitels «Wirkungsart» nicht ohne weiteres klar, daß Hufeland eine ziemlich einfache Erklärung für die vielfältigen Wirkungen des Opiums gibt, diese aber immer wieder in andere Formulierungen kleidet. Das Opium enthält nach Hufeland zwei Stoffe: einen, der dämpfend auf das Nervensystem wirkt, und einen, der erregend auf das Herz und Blutsystem wirkt. Obwohl er somit zwei unterschiedliche Stoffe im Opium postuliert, lehnt er trotzdem die chemische Analyse ab und schreibt die volle Wirkung nur dem Ganzen zu.

\subsection{Indikationen der Opiumtherapie}

Bei den Indikationen zur Opiumtherapie stützt sich Hufeland auf seine Theorie der zwei verschieden wirkenden Komponenten des Opiums. Die «Grundindikation» ist: «Krampf, Nervosität, d.h. anomalische oder erhöhte Sensibilität, aber, wohl zu merken, bei herabgestimmter Lebensenergie des Blutsystems und des ganzen Blutlebens» (S. 833). Wo die Herztätigkeit gesteigert ist, allgemeine Blutfülle (Plethora) oder Entzündungsbereitschaft deutlich sind, ist Opium nicht am Platz. «[...] mit andern Worten: je mehr wahre Lebensschwäche vorhanden ist, desto mehr ist das Opium angezeigt und passend; [...] je mehr durch vorhergegangenen Säfteverlust oder durch künstliche Blutentziehung und gastrische Ausleerungen der Körper geschwächt ist, desto besser bekommt das Opium» (S.833). Mit diesen Erklärungen bewegt sich Hufeland im Brown'schen System: Leben ist Erregung; Krankheit entsteht durch übermäßig gesteigerte oder verminderte Erregung, zu große oder zu geringe Lebenskraft, Sthenie oder Asthenie. Hufeland warnt vor der Anwendung des Opiums, «so lange noch eine Blutentziehung angezeigt ist». - «Durch solchen unzeitigen Gebrauch des Opiums kann jedes Fieber in einen Typhus verwandelt werden»-also in einen Krankheitszustand mit Benommenheit, Verwirrtheit und anderen zerebralen Symptomen (S.833f.)

Die Indikationen, die heute am ehesten anerkannt würden, erwähnt Hufeland eher beiläufig: «Speciell und ganz besonders indicirt ist es: bei Schmerzen, bei dem Bedürfniß Schweiß zu erregen, überhaupt wenn es nöthig ist, einen starken Antrieb vom Centrum nach der Peripherie hervorzubringen, bei schwächenden Darmausleerungen» (S. 833). Die Anwendung 
von Opium bei Schmerz und bei Durchfällen ist auch heute noch therapeutisch vertretbar, falls man keine neueren Medikamente, die nicht so stark süchtigmachend sind, zur Verfügung hat.

Es folgt nun eine Übersicht über die wichtigsten Krankheiten, bei denen das Opium angewendet werden kann (S.834 ff.). Hufeland empfiehlt Opium mit den nötigen Kautelen auch bei «Lokalentzündungen», vor allem bei Pleuritis, Herzentzündung, Entzündungen der Unterleibseingeweide, Cholera acutissima und orientalis, Ileus inflammatorius, Blasenentzündung, Halsentzündung, Croup, Hirnentzündung und Augenentzündung (S. $834 \mathrm{ff}$.).

Als Hauptkriterium für die Anwendung des Opiums bei Nervenfieber und Typhus (was im wesentlichen ein und dasselbe ist) erwähnt Hufeland das Fehlen von Blutkongestion und Entzündung (S.838ff.). Zum Beispiel, «wenn von Anfang an das Nervenfieber rein nervös, d.h. ein Schwachheitsfieber, ist, durch übermäßige Anstrengung der Kräfte, Excesse in Venere und Onanie, anhaltendes Übermaaß im Trinken u. dgl., erzeugt, oder in einem schon nervösen Subjekte entstanden ist, wenn gar kein Zeichen von Entzündung sich äußert. [...]-Dahin gehört auch die neulich so gerühmte Anwendung des Opiums bei der Trunksucht, bei dem Delirium potatorum.»

Hufeland zitiert folgenden Fall von «Typhus», bei dem er Opium angewendet hat. «Nie werde ich die Freude vergessen, die mir hier das Opium bei einem meiner werthesten Herren Kollegen bewirkte. Er lag am 7ten Tage eines sehr heftigen Typhus, mit kleinem kaum zu zählendem Puls, Sopor, Delirien, Flechsenspringen. Blutentziehungen, Kälte, Abführungen, Calomel, waren reichlich angewendet. Er erhielt obige Pulver mit Calomel und Opium, und nach 6 Dosen war der Puls langsam und gehoben, die Krampfzufälle verschwunden, der Kopf frei und die Krise bewirkt; die Besserung fing von dem Tage an sich einzustellen und vollkommen zu machen. - Und wie viele ähnliche Fälle könnte ich anführen!» (S. 840) Sechs Dosen des verwendeten Pulvers enthalten 2 Gran, d.h. 0,12 g Opium.

Beim «Wechselfieber», vor allem beim «perniciösen Wechselfieber», einer Malaria-Form, wo jeder Anfall mit einem «lebensgefährlichen Symptom, Apoplexie, Sopor u. dgl. verbunden ist», glaubt Hufeland an einen Krampf als Ursache und befürwortet ebenfalls den Gebrauch des Opiums (S.841).

Bei Krämpfen rühmt er seine besonders gute Wirksamkeit, klagt aber gleichzeitig darüber, daß die Ärzte nur noch Opium verwenden und nicht mehr zwischen den verschiedenen Krampfarten mit ihren unterschiedlichen 
therapeutischen Indikationen zu unterscheiden wissen (S. $841 \mathrm{ff}$.). Wie beim Nervenfieber und Typhus unterscheidet Hufeland zwischen «rein nervösen» Krämpfen und Krämpfen mit «Vollblütigkeit, Kopfkongestion, oder gar entzündlicher Diathesis». Als dritte Kategorie nennt er hier noch «gastrische Unreinigkeiten». Die Vollblütigkeit muß zuerst durch Aderlässe, die gastrischen Unreinigkeiten durch Brechmittel beseitigt werden, ehe die Krämpfe, wie bei rein nervöser Genese, mit Opium kuriert werden können.

Auch postoperativ und posttraumatisch verwendet Hufeland das Opium (S.842ff.), wobei er aber nicht den Schmerz als Hauptindikation nennt, sondern einen Zustand, wo «der Kranke krampfhaft, starr, halb leblos daliegt», ohne klare Entzündungszeichen, mit einer Eiterung, die «mehr ichorös als purulent» ist. Beim Trismus und Tetanus posttraumaticus ist Opium das «einzige übrige Rettungsmittel. Die ganze medicinische Welt ist davon überzeugt» (S. 843).

Beim Wahnsinn unterscheidet Hufeland wieder zwischen der «rein nervösen» und der «plethorischen» Form mit «aktiv phlogistischer Aufregung des Gehirns». - «[...] wo der ursprüngliche Grund des Übels nicht im Gehirn und Nervensystem, sondern im Unterleibe in Anhäufungen, Überfüllungen, Stockungen der Präkordialeingeweide liegt, wie dies besonders bei der melancholischen Geistesverstimmung der Fall ist, da wird Opium nur schaden» (S. 844). In dieser pathogenetischen Auffassung der Melancholie zeigt sich deutlich Hufelands Verbundenheit mit der alten Humoralpathologie $^{13}$. Auch hier wendet Hufeland wieder das gleiche Behandlungsschema an, indem er «nach gehobener materieller Ursache, wenn nur das Übel als rein nervöser Zustand fortdauert», Opium anwendet.

Selbst beim Diabetes verordnet Hufeland Opium (S.846), falls nicht noch «entfernte Ursachen, als Plethora, Kongestion, Metastasen, Abdominalverstopfung» zuerst beseitigt werden müssen. Er erklärt den Diabetes mellitus als «krankhaften Erethismus [Erregungszustand] der Nieren, der [...] mit einer eigenthümlichen Abalienation der Produktivität (chemischorganischen Sekretionsproceß) verbunden ist, so daß statt der gewöhnlichen Harnsalze sich Zucker erzeugt». Das Opium, sein «Hauptmittel», bewirkt «eine Aufhebung des örtlichen Nervenerethismus und kräftige peripherische Impulsion» mit gesteigerter Schweißabsonderung.

Auch bei der Syphilis behebt das Opium die Symptome der Krankheit, tötet aber im Gegensatz zum Quecksilber das «syphilitische Miasma» nicht. Hufeland schlägt deshalb eine Kombinationstherapie mit Quecksilber und Opium vor (S.847ff.). Sie hat den weiteren Vorteil, daß der merkurielle 
Speichelfluß durch das Opium vermindert wird. Überhaupt ist Opium ein Antidot bei Metallvergiftungen, namentlich durch Blei, Quecksilber und Arsen.

Zur Stillung des furchtbaren Hautreizes wie zur Förderung der Eiterung verwendet Hufeland das Opium bei den Pocken und will so die Rettung vieler Kranken bewirkt haben (S.850 ff.). Auch die schweißtreibende Wirkung des Opiums wirkt bisweilen Wunder (S. 851): «Ein Mann von mittleren Jahren litt schon Jahre lang an einem Hüftweh, was ihn hinken machte. Er bekam einen Anfall von Ruhr, der ihn zum Gebrauch des Opiums nöthigte. Die Folge war ein sehr starker Schweiß und ein allgemeiner Hautausschlag, und mit diesem war sein Hüftübel gehoben, und er seitdem völlig frei im Gebrauch seiner Füße. - Hier war wahrscheinlich das Hüftübel nichts als eine Metastase eines rheumatischen Stoffs auf die Hüfte. Dieser wurde durch das Opium mobil gemacht und durch die Hautkrise in der Form des Exanthems ausgeschieden.»

Nach alledem verwundert es kaum mehr, daß Hufeland auch gutartige Gewächse, «Pseudorganisationen» wie Polypen der Vagina, der Nase usw. durch Auftragen von Opium zum Schwinden gebracht hat (S.851). Diese «örtliche Heilkraft» scheint uns außerhalb der von Hufeland sonst streng beachteten Doppelwirkung des Opiums auf Nerven und Blut zu stehen.

Ungetrübt tritt Hufelands Menschlichkeit bei der letzten seiner OpiumIndikationen hervor, bei der «Palliation» (S.852f.). Da spricht er nun über «die palliative Kraft des Opiums, die man gewöhnlich als Nebensache betrachtet, die aber oft die Hauptsache wird [...]. Sie heißt, Linderung der Leiden und Schmerzen, Beruhigung, Erhebung des Gemüths und Erleichterung des Sterbens.»

«Kein Mittel unter allen vermag in solchem Grad Schmerz und Angst zu lindern, ja auf eine Zeitlang ganz wegzuzaubern, als dieses. Nicht hundert, sondern tausend Male habe ich meine Kranken am andern Morgen völlig verwandelt gesehen, schon in Miene, Sprache und ganzem Ausdruck, wenn sie Abends vorher Opium genommen hatten, und gewöhnlich 24 Stunden dauert die Wirkung.

Ich will nur an die trostlose Lage des allmählig unter Beängstigung und Luftmangel dahin schwindenden unheilbaren Lungensüchtigen, an die grausamen, Tag und Nacht quälenden Schmerzen des rettungslosen Krebskranken, an die lange Todesangst des Brustwassersüchtigen, erinnern. - Wer möchte da Arzt sein ohne Opium? Wie viele Kranke sind nicht dadurch schon der Verweiflung entzogen worden! Denn das ist das Große dieses Mittels, daß es nicht blos die körperlichen Schmerzen und Beschwerden besänftigt, sondern auch der Seele eine ganz eigne Kraft, Erhebung und innere Beruhigung gewährt.» 


\subsection{Dosierung und Überdosierung}

Hufeland ist sich des Problems der Dosierung und der Überdosierung bewußt. So schreibt er im Anhang zum «Enchiridion medicum» im Kapitel "Verhältniß [des Arztes] zu den Kranken»: Es ist schrecklich, Flaschen mit halben und ganzen Unzen [29,8 g] Opium in den Krankenstuben stehen zu sehen; und geschieht auf diese Weise ein Unglück, so ist es allemal die Schuld des Arztes» (S.901 f.).

Prüft man nun aber die Stellen im Hauptteil des «Enchiridion medicum», an denen Hufeland den Gebrauch des Opiums für bestimmte Krankheiten empfiehlt, so findet man bei insgesamt 89 Stellen nur zwölfmal eine Dosisangabe. Die Dosisangaben sind nur schon deswegen oft ungenau, weil Hufeland ganz verschiedene Präparate benutzt: Opium (S.107, 143, 145, 284, 288, 377), Tinctura opii (S.142, 700, 724), Extractum opii aquosum (S. 199), Laudanum Sydenhami S.499) und Doversches Pulver (S. 836). Der Gehalt an Morphium war von Präparat zu Präparat und von Hersteller zu Hersteller verschieden - eine Tatsache, die von anderen Autoren jener Zeit bereits warnend erwähnt wurde.

Hufeland weist speziell darauf hin, daß bei Kindern besonders vorsichtig dosiert werden muß, wobei er das Opium vor allem bei Durchfällen benützt und zwischen ${ }^{1 / 60}$ bis zu ${ }^{1 / 40}$ Gran $(1-1,5 \mathrm{mg})$ Tinctura opii oder Opium gibt (S.724, 737). Die Gefahren der Opiumtherapie bei stillenden Müttern erwähnt er nicht.

Es war Hufeland wahrscheinlich nicht ganz klar, daß Opium bei äußerlicher Anwendung als Pflaster oder Einreibung, aber auch in der Form von Klistieren nach lokaler Resorption systemisch wirkt. Im «Enchiridion medicum» werden Opiumpflaster, Opiumeinreibungen und Opiumklistiere dreizehnmal erwähnt, mit einer einzigen Warnung vor einer Überdosierung bei folgender Anwendungsweise zur Therapie einer örtlichen Lähmung: "Auch die endermatische Methode verdient Benutzung. Sie besteht darin, daß man eine kleine Hautstelle durch ein Blasenpflaster entblößt, und nun auf die rohe Oberfläche ein Exutans ${ }^{14}$ applicirt, am besten Extr. Nuc. Vomic. oder Morphium, Belladonna u. dgl., aber auch hier mit großer Vorsicht in den Dosen, weil hier die Mittel oft stärker wirken, als innerlich gegeben. Am besten wählt man eine Stelle am Ursprung des gelähmten Nerven» (S. 356). Hier wird ausnahmsweise ausdrücklich das Morphium erwähnt.

An einer anderen Stelle hingegen (S. 724) empfiehlt Hufeland bei Kindern den Gebrauch des Opiums «lieber nur äußerlich und in Klistieren» und rät 
allgemein zur «Anwendung der Heilmittel durch die Haut ...; denn die Haut hat hier sowohl mehr Nervenempfindlichkeit, als auch mehr Resorptionskraft, und man vermeidet dadurch manche nachtheilige Einwirkungen auf den Magen und Darmkanal, z. B. bei dem Opium».

Schließlich war Hufeland davon überzeugt, daß das Opium bei lokaler Anwendung seine beruhigende und schmerzstillende Kraft unmittelbar den Nerven mitteilt. Die andere Wirkungskomponente, nämlich die Stimulation des Kreislaufs und des «Blutlebens», bleibt seiner Meinung nach dabei völlig stumm (S. 828):

«Ferner die örtliche sedative Wirkung des Opiums; es wirkt, auch äußerlich aufgelegt, schmerzstillend und besänftigend, ohne alle Einwirkung oder Aufregung des Gefäßsystems, ja es kann - wie ich selbst erfahren habe, als ich an einem heißen Tage ein beträchtliches Stück Opium lange in der Hand gehalten hatte - bei einer starken und anhaltenden äußeren Applikation, ohne alle Blutaufregung, blos durch den Nervenkonsensus, auf das Sensorium wirken und narkotische Zufälle erzeugen. Das bloße Auflegen auf die Schläfe macht Schlaf. Die örtliche Applikation auf den Magen, beim Einnehmen, hebt unmittelbar die örtliche Empfindlichkeit und Nerventhätigkeit dieses Organs (Appetit, Verdauungskraft und peristaltische Bewegung) auf; die örtliche Anwendung auf den Darmkanal in Klystieren die peristaltische Thätigkeit des Darmkanals.»

Aus alledem ergibt sich, daß Hufeland die Anwendung des Opiums in Pflastern und Klistieren für weniger gefährlich hält als die Einnahme per os. Genaue Dosis-Angaben, die uns die verwendete Morphinmenge errechnen ließen, fehlen in seinem Buch.

\subsection{Gewöhnung und Sucht}

Im ersten Teil des «Enchiridion medicum» nennt Hufeland das Opium als Ursache geistigen und körperlichen Zerfalls. Es kann Wahnsinn (S.254), Tabes (S.403) und «Darrsucht», d.h. Schwindsucht der Kinder (S. 755) bewirken.

In einer Anmerkung (S.822) versucht Hufeland die unterschiedliche Wirkung des Opiums bei «nicht daran gewöhnten» und bei «gewöhnten» Personen zu erklären. Er erwähnt Türken und Orientalen und spricht vom «tollen Muth» der Türken in der Schlacht unter Opiumeinwirkung. Er erwähnt auch einheimische Personen, «die sich durch langen und starken Gebrauch des Opiums verwöhnt haben, dies Mittel in den stärksten Gaben nehmen, ohne einen anderen bemerkbaren Effekt als Vergessenheit seiner selbst, seiner Schmerzen, seines ganzen kranken Zustandes, und dadurch entstehende Heiterkeit und Zufriedenheit». 
Im Abschnitt «Nachtheile und Gefahren» beschreibt Hufeland als letzten Punkt die «Verwöhnung» mit Opium (S. 854):

«Man kann sich, bei langwierigen Übeln, zuletzt dergestalt an den Gebrauch des Opiums gewöhnen, daß es tägliches Bedürfnis wird, auch nach gehobenem Leiden, zur Erhebung des Gemeingefühls auf den Punkt des Wohlseins, der Lebendigkeit, der physischen und geistigen Brauchbarkeit, - ganz auf die nämliche Weise, wie sich der Branntweintrinker zuletzt an den Branntwein gewöhnt und er ihm zuletzt zum unentbehrlichen Bedürfniß wird - aber auch mit der nämlichen Folge, dem Bedürfniß immer höherer Gaben, - die Opiumsucht - ganz analog der Trunksucht und ihren Wirkungen, immer größerer Nervenschwächung, Zittern, Zerstörung der Verdauungs- und Reproduktionskraft, zuletzt Delirium tremens, Stumpfheit der Sinne und des Geistes, Blutungen, Auflösung des Blutes, Tabes».

Dieser Passus zeigt, daß Hufeland die Analogie zwischen Trunksucht und Opiumsucht erkannte; der letzteren hat er, wie Erich Hintzsche feststellte, als erster ihren Namen gegeben ${ }^{15}$.

\section{Darstellung der Opiumtherapie in den zum Vergleich benützten Nachschlagewerken}

Die konsultierten Werke sind im Abschnitt 2, «Quellen», vorgestellt worden. Zwei dieser Texte sind vor dem «Enchiridion medicum» erschienen, zwei danach.

\subsection{Dictionaire des sciences médicales, Bd.37, Paris 1819}

Die Bedeutung des Opiums wird auch im «Dictionaire des sciences médicales», von Mérat hervorgehoben: «... c’est, de tous les médicaments, le plus héroique et celui dont la médecine peut le moins se passer» (S. 482).

Sonst aber gibt es kaum eine Übereinstimmung zwischen Hufeland und Mérat. Die chemische Analyse des Opiums wird im «Dictionaire» ausführlich geschildert und gewürdigt; das Morphin gilt als wesentlichste Komponente des Opiums: «C'est à la présence de la morphine dans l'opium, que paraissent dus ses effets, quoiqu'on ignore encore si c'est le seul principe actif» (S.472). 
Die Opiumwirkung wird wie folgt charakterisiert (S.474ff.): Es wirkt schlafbringend, vermindert die Schmerzempfindung des Gehirns, führt bei großen Dosen zu Brechreiz und verlangsamt die Verdauung. Auch wird eine Wirkung auf die Kapillaren beschrieben, die zu einer Stase des Blutes und somit zu einer Entzündung führe.

In diesem Zusammenhang geht Mérat auf die Kontroverse ein, die über die Frage entbrannte, ob das Opium - im Sinne von John Brown's Erregungsmedizin ${ }^{16}$ unter die erregenden oder die dämpfenden Mittel einzureihen sei. Hufeland löste dieses Problem bekanntlich, indem er das Opium als die «Verbindung eines narkotischen Stoffs mit einem exitierenden» beschrieb (siehe oben S. 103). Mérat dagegen faßt das Opium als rein dämpfend auf. Die Erweiterung der Kapillaren und damit die Stase des Blutes sind Dämpfungseffekte, die Erregung des Herzens und des Kreislaufes nur eine Reaktion darauf (S.476ff.).

Als Hauptindikation für den Gebrauch des Opiums gelten im «Dictionaire» Schlaflosigkeit und Schmerz (S.485). Daß das Opium keine Krankheiten heilt, sondern nur deren Symptome bekämpft, wird betont: «L'opium n'est point un médicament qui guérisse par une vertu particulière, ce n'est le spécifique d'aucune affection; c'est seulement, en stupéfiant certains symptômes, qu'il allège leur intensité, qu'il adoucit les maladies, et leur permet de parcourir leurs périodes avec plus de facilité» (S.483). Das Opium ist das Spezifikum gegen den Schmerz: «L'opium est véritablement le remède de la douleur, le spécifique de ce symptôme (S.485).

Der «Dictionaire des sciences médicales» gibt allgemeine Richtlinien für die Dosierung (S.479ff.). Kleine Dosen, ein halbes bis ein ganzes Gran, $(0,026-0,053 \mathrm{~g})^{17}$, wie sie in der Medizin meist gebraucht werden, beruhigen, sind schlafbringend und erleichtern die Schmerzen. Große Dosen bewirken einen «Narkotismus», d.h. einen pathologischen Schlafzustand, wobei die Empfindlichkeit individuell sehr verschieden ist. Exzessive Dosen von mehr als 8-15 Gran (0,42 bis 0,9 g) rufen Vergiftungserscheinungen hervor, die zum Tode führen. Mérat rät dem Arzt, ein einziges Opiumpräparat zu benützen, um Dosierungsfehler zu vermeiden.

Sehr wichtig ist, daß im «Dictionaire des sciences médicales» auf die Resorption des Opiums bei sogenannt äußerlicher Anwendung hingewiesen wird: bei Opiumpflastern und -verbänden, bei Opiumklistieren und bei Opiumbädern kann es zu allgemeiner Wirkung, ja zur Vergiftung kommen. Auch die im Verlauf der Behandlung eintretende Opiumtoleranz, die Abhängigkeit und die Entzugssymptome werden beschrieben (S.480 ff.). 
Auch die «Encyclopädie der medicinischen Wissenschaften» hält den hohen Rang der Opiumtherapie fest: «[...] es gibt kein berühmteres Heilmittel und keines, über welches man so viel geschrieben hätte, als über das Opium» (A. Richard, S.301). Guersant erklärt (S.310), «daß man auf die Ausübung der Kunst Verzicht leisten müßte, wenn das Opium nicht vorhanden wäre».

Die chemische Analyse des Opiums wird in der Encyclopädie klar dargestellt (Richard, S.302ff.) und seine Eigenschaften, wie schon im «Dictionaire» von 1819, hauptsächlich dem Morphin zugeordnet (Guersant, S.305).

Die Wirkungen und Begleiterscheinungen der Opiumtherapie wie Mundtrockenheit, Verstopfung, Schlaf, Miosis, Verlangsamung der Atemfrequenz, Abnahme des Hustenreizes, Schweiß werden nüchtern aufgezählt, ohne den Versuch, sie unbedingt zu erklären. Die Wirkungsweise des Opiums ist nach Guersant unbekannt (S.304 ff.).

Als Indikationen der Opiumtherapie gelten alle sehr schmerzhaften Krankheiten, Palliation bei unheilbaren Leiden, Dysenterie, Cholera, Diarrhoe und Hustenreiz (S. $308 \mathrm{f}$.).

Man findet auch Dosierungshinweise. So werden Morphinsalze in einer Dosis von einem halben bis zwei Gran $(0,03$ bis $0,12 \mathrm{~g})$ gegeben, wobei wäßriger Opiumextrakt dreimal schwächer als Morphiumsalz ist. Dagegen sind die Hinweise auf Opiumsucht und -gewöhnung dürftig und beschränken sich auf Abmagerung, Mattigkeit und «Verkümmerung» bei langdauernder Opiumtherapie (S.304).

\subsection{Dictionnaire de médecine, 2. Auflage, Bd.22, Paris 1840}

Der therapeutische Wert des Opiums wird im «Dictionnaire de médecine» nicht mehr so hoch herausgehoben wie in den beiden älteren Werken. Die chemische Analyse des Opiums und die Bedeutung seiner Einzelsubstanzen werden dagegen ausführlich besprochen (Soubeiran, S. $212 \mathrm{ff}$.). Soubeiran betont, daß das Morphin die eigentliche Wirksubstanz ist (S. 224). Daneben werden Codein und Narkotin erwähnt.

Die Symptome der Opiumwirkung werden ausführlich aufgezählt: Mundtrockenheit, Appetitverlust, Durst, Erbrechen, Verstopfung, Schläfrigkeit, Schmerzfreiheit, Miosis, Verlangsamung der Atemfrequenz, Minderung des Hustenreizes, Schwitzen, Harnverhaltung (Guersant, S.227 ff.). 
Guersant versucht jetzt, diese Symptome in drei Hauptgruppen zu ordnen, und unterscheidet: 1) eine schlafbringende, sensibilitätsvermindernde und schmerzstillende Eigenschaft; 2) eine sekretionsvermindernde Eigenschaft, die vor allem auf Schleimhäute wirkt; 3) eine den Kapillardruck bestimmende Eigenschaft, die zum Schwitzen führt (S.244). Als paradoxer Effekt der Opiumtherapie wird der ausgesprochene Erregungszustand erwähnt, der unerwartet auftreten kann (S.244f.). Als Zeichen der Überdosierung nennt Blache (S.257ff.) Stupor und Konvulsionen oder aber Muskelerschlaffung. In mäßig toxischer Dosis ruft das Opium beim Menschen Schwindel, gelegentlich aber auch Euphorie hervor, «cette action exhilarante si recherchée par les Orientaux». Bei höherer Dosis zeigt sich die Vergiftung durch Übelkeit an, es folgen allgemeine Erschlaffung und Somnolenz bis zum Koma. All das wird genau beschrieben, unter Einbeziehung tierexperimenteller Beobachtungen, auch für die Neben-Alkaloide Codein und Narkotin.

Genau wie in der «Encyclopädie der medicinischen Wissenschaften» einige Jahre zuvor, gibt es auch jetzt für Guersant keine haltbare Erklärung für die Wirkungsweise des Opiums: «les explications plus ou moins ingénieuses qu'on a données jusqu'ici de ces phénomènes n'ont pas beaucoup servi à éclairer la cause première de l'action narcotique, et le quare opium facit dormire est toujours tout aussi obscur. Nous ne savons réellement rien sur la cause somnifère de l'opium [...]» (S. 245).

Die Indikationen zur Opiumtherapie werden nach Organsystemen aufgeteilt (Guersant, S.247ff.). In der Gastroenteorologie gelten nervöses Erbrechen, Cholera, Dysenterie, Durchfälle sogar bei Kindern als Indikationen. Bei den Lungenkrankheiten wird die Dämpfung des Hustenreizes angestrebt, bei chronischer Bronchitis und Phthisis ist jedoch die Opiumtherapie umstritten. Bei allen schmerzhaften Krankheiten und Operationen wird Opium gegeben. Rheumatismus, Tetanus, Chorea, Delirium tremens und senile Gangrän sind weitere Indikationen.

Soubeiran weist im «Dictionnaire de médecine» auf den unterschiedlichen Morphingehalt der einzelnen Opiumsorten (S.212) und Opiumpräparate (S.219) hin. Kernstück der Dosierungsangaben ist eine zweiseitige Tabelle, in der der Morphingehalt der verschiedenen Präparate mit kristallisiertem Morphin verglichen wird und wo die Gewichte fortschrittlicherweise sowohl in Gramm wie in Gran (0,0531 g) angegeben werden (S.225 f.). Als Standarddosis gelten 25 bis 100 Milligramm Opium entsprechend $1 / 2$ bis 2 Gran (Guersant, S.227). 
Als Letaldosis gelten 4 bis 60 Gran $(0,21$ bis 3,18 g) Opium. Falls der Patient die ersten zwölf Stunden nach Einnahme der Überdosis überlebt, hat er gute Chancen, sich von der Vergiftung zu erholen. Es werden auch Vorschläge zur Therapie der Vergiftung gemacht, wobei vor allem Emetika und Magenspülung empfohlen werden. Auch die künstliche Beatmung wird erwähnt (Blache, S. 259 ff.).

Wie schon im «Dictionaire des sciences médicales» (siehe oben S.111), wird auch im «Dictionnaire de médecine» vor der sogenannt äußerlichen Anwendung des Opiums gewarnt. Auf tödliche Vergiftungen bei Kindern und Erwachsenen durch Opiumpflaster (Guersant, S.241 ff.) und Opiumklistiere (S.227, 243) wird ausdrücklich hingewiesen. Die endermatische Methode, bei der Opiumpflaster auf die offene Haut gelegt werden (vgl. oben S. 104), wird wegen ihrer relativ sicheren systematischen Opiumresorption empfohlen, falls das Medikament nicht per os gegeben werden kann (S. 242).

Die Opiumabhängigkeit und -gewöhnung werden nur ganz kurz erwähnt (Guersant, S.229f.).

\subsection{Universal-Lexicon der practischen Medizin und Chirurgie,} Bd.10, Leipzig 1841

Die Bedeutung des Opiums für die Medizin kommt hier noch in dem beträchtlichen Umfang des Artikels zum Ausdruck: er umfaßt 98 Spalten (S.134/2-183/1). Die chemische Analyse wird eingehend behandelt; als wichtigste der 18-20 bekannten Bestandteile werden die «stickstoffhaltigen Alkaloide» Morphin, Codein, Narcotin und Narcein sowie das Meconin genannt (S. 137). Es folgt die Beschreibung der offiziellen Präparate, darauf die Toxikologie einschließlich der Therapie der Vergiftung.

In diesem Abschnitt werden rein deskriptiv Opiumrausch und -sucht dargestellt (S.153 f., Zitate nach K.G. Neumann): «So ist es denn begreiflich, wie die Menschen sich an den Rausch des Opiums leidenschaftlich gewöhnen können, da es heitere Delirien und Träume erregt und weder Erbrechen und Durchfall, noch Kopfschmerz hinterläßt, wie der Rausch von geistigen Getränken.» Neumann betont die besondere Empfindlichkeit der Kinder und Säuglinge: «Wenn stillende Frauen Opium nehmen, verfallen die Säuglinge in Narcose, während die Frauen selbst nicht die mindeste fühlen. Im Ganzen: je jünger die Menschen, desto weniger Opium vertragen sie; haben sie es aber einmal genommen, so wirkt jede nachfolgende Gabe weniger als die vorhergehende [...]» Der Ausdruck «Sucht» taucht hier nicht auf; die Opium- 
Abhängigkeit wird weder als Krankheit gewertet noch moralisch verurteilt. Martin-Solon dagegen setzt die chronische Schädigung der orientalischen Opium-Esser (frühzeitige Stumpfheit und auffallende Schwäche) in Parallele zur gleichartigen Veränderung «unserer Trunkenbolde» (S. 161).

Die praktische Seite, der therapeutische Gebrauch des Opiums macht den Schluß. Daß die Ärzte seit dem Altertum ununterbrochen das Opium brauchten, beweist für Martin-Solon mehr als alles andere «den unwiderlegbaren Nutzen, den diese Substanz in Krankheiten gewährt» (S.159). Die Anwendung wird erst systematisch nach Präparaten abgehandelt (mit Angaben zur Dosierung), dann folgen die Indikationen - kritisch beleuchtet - für den äußerlichen und den innerlichen Gebrauch, schließlich die Kontraindikationen. Die Schmerzbekämpfung steht an erster Stelle; die ganze Liste der diskutierten und dabei oft in Frage gestellten oder relativierten Indikationen ist noch fast so umfangreich wie bei Hufeland. Sie umfaßt für den innerlichen Gebrauch: 1. Schmerzen, 2. Fieber, 3. Entzündungen, 4. Hämorrhagien, 5. Wassersuchten, Schleim-, Bauch- und andere Flüsse, 6. Nervenkrankheiten («Nevrosen»), 7. «organisierte Verletzungen», d.h. Gangrän, Tuberkulose u. a. Auch psychiatrische und geburtshilfliche Indikationen werden erörtert. Die Darstellung läßt auf große Meinungsunterschiede unter den praktizierenden Ärzten schließen. Damit stimmt MartinSolons abschließende Feststellung überein, «daß, wie häufig auch die Anwendung des Opiums seyn mag, doch die eigentlichen Indicationen zu seinem Gebrauche noch lange nicht gehörig festgesetzt sind» (S. 178).

Ein mit «M.» gezeichneter Anhang (S. 178-183) gibt die homöopathische Opiumanwendung wieder: extrem weites Indikationsspektrum bei praktisch vollkommener Auflösung der Substanz, nämlich «Verdünnung bis zur

decillionfachen Kraftentwicklung». Eine Dezillion ist eine Eins mit 60 Nullen - in einer solchen Verdünnung von $1: 10^{60}$ ist das Opium auf jeden Fall absolut unschädlich!

\section{Schlußfolgerungen}

Im zeitgenössischen Vergleich fällt besonders Hufelands Geringschätzung der chemischen Analyse des Opiums auf; die vier Nachschlagewerke, die wir beigezogen haben, gehen alle sorgfältig darauf ein und schreiben die Opiumwirkung im wesentlichen dem Morphin zu. Das ermöglicht auch wesentlich präzisere Dosierungsangaben, als man sie bei Hufeland findet. 
Die Verfasser dieser Artikel gestehen sich auch ein, daß sie nicht erklären können, wie das Morphin und die übrigen Komponenten des Opiums (Codein u. a.) wirken. Hufeland dagegen schwelgt in vitalistischen Erklärungen (s. Abschnitt 3.2.), wie sie in der deutschen naturphilosophischen Medizin des frühen 19. Jahrhunderts beliebt waren. Zum Teil reichen sie historisch auch noch weiter zurück, zunächst zu John Brown (so etwa der Gegensatz von «exzitierender» und «sedativer» Kraft). Derartige Vorstellungen und Begriffe, wie die «Lebenskraft» und die von ihr abgeleiteten besonderen vitalen Kräfte oder die altehrwürdige «Sympathie», wurden von den Medizinern und Pharmazeuten jüngerer Generationen, vor allem in Frankreich, nicht mehr als wissenschaftliche Erklärungen anerkannt. Als einen deutschen Kritiker jener spekulativen medizinischen Theorien nennen wir Carl August Wunderlich (1815-1877), der nun freilich mehr als fünfzig Jahre jünger als Hufeland war ${ }^{18}$. Doch selbst ein älterer Zeitgenosse wie Samuel Gottlieb Vogel (1750-1837) war, wie Jonas Landmann (s. Anm.3) gezeigt hat, im Gegensatz zu Hufeland durchaus bereit und fähig, in seine ärztliche Diagnostik die neuen französischen Fortschritte wie Perkussion und Auskultation zu integrieren. Hufelands Rückständigkeit gegenüber der wissenschaftlichen Entwicklung in der Medizin des frühen 19. Jahrhunderts läßt sich nicht ausschließlich durch sein Alter erklären (74 Jahre bei der Publikation des «Enchiridion»); sie wurzelt auch in seiner Persönlichkeit. Bei dem hohen Ansehen, das er als ärztlicher Mentor genoß und das sich in der Verbreitung seines «Enchiridion medicum» zeigt (vgl. Abschnitt 2), konnte sich seine wissenschaftlich konservative Einstellung jedoch nur nachteilig auf das Niveau der ärztlichen Praxis, vor allem im deutschen Sprachgebiet, auswirken.

Hufelands mangelnde Naturwissenschaftlichkeit charakterisiert auch seine Opiumtherapie: er empfiehlt verschiedene Zubereitungen mit wechselndem Opium- und Morphingehalt nebeneinander; seine Dosierungsangaben sind schon deshalb unpräzis. Der Indikationsbereich des Opiums richtet sich bei Hufeland nach Tradition und Erfahrung, auch nach seiner Überzeugung, daß es in der ganzen Medizin neben Aderlaß und Brechmitteln nichts gebe, was dem Opium an Kraft und vielseitiger Verwendbarkeit gleichkomme. Die französischen Autoren der Nachschlagewerke von 1819, 1832 und 1840 sind hierin zurückhaltender und präziser; sie benutzen Opium resp. Morphin vor allem als Schmerzmittel. Das auf deutschen wie französischen Autoritäten aufbauende «Universal-Lexicon» von 1841 macht es hingegen deutlich, daß die Grenzen für die Therapie mit Opiaten in der damaligen 
ärztlichen Praxis wirklich sehr weit, unbestimmt und kontrovers waren. Hufelands Vorliebe für das Opium bewahrt ihn übrigens vor der verhängnisvollen Überschätzung und Überdosierung des Quecksilbers, der manche Ärzte seiner Zeit huldigten ${ }^{19}$.

Hufeland und seine Jünger mögen die Opiate auch deswegen manchmal überdosiert haben, weil sie deren Resorption durch die intakte Haut und aus den unteren Darmabschnitten unterschätzten; für Hufeland war das Opium-Klistier eine äußerliche Anwendung von geringer systemischer Wirkung. Ganz allgemein ist Hufeland sich jedoch der Gefahr der Überdosierung bewußt und schätzt sie vor allem bei Kindern mit Recht hoch ein. Doch erst die beiden nach seinem Tod erschienenen Nachschlagewerke gehen gründlich auf die Toxikologie der Opiate ein.

Hufeland prägt dagegen den Ausdruck «Opiumsucht» für den Zustand der Abhängigkeit, der durch «Verwöhnung» mit dem Mittel entsteht. Die Beobachtung der Abhängigkeit war damals zwar nichts Neues; Mérat beschreibt sie im «Dictionaire» 1819 ebenfalls. Im Universal-Lexicon wird sie 1841 wie in früheren Jahrhunderten ${ }^{20}$ als Eigenheit der Orientalen dargestellt; die «Encyclopädie» 1832 und der damit verwandte «Dictionnaire» 1840 gehen nicht darauf ein.

An wissenschaftlichem Niveau stehen die Opium-Artikel der französischen Autoren in den Nachschlagewerken von 1819, 1832 und 1840 über Hufelands «Enchiridion medicum» von 1836. Bei Hufeland tritt jedoch noch ein anderes Element der ärztlichen Behandlung ans Licht: das Eingehen auf den ganzen Menschen - bis zur Erleichterung des Sterbens, der «Uthanasia» im richtigen, alten Sinn.

\section{Anmerkungen}

${ }^{1}$ Ch. W. Hufeland, Enchiridion medicum oder Anleitung zur medizinischen Praxis. Vermächtniß einer funfzigjährigen Erfahrung. Berlin 1836.

${ }^{2}$ F.W.A.Sertürner (1783-1841). Darstellung der reinen Mohnsäure (Opiumsäure); nebst einer chemischen Untersuchung des Opiums, mit vorzüglicher Hinsicht auf einen darin neu entdeckten Stoff. J. Pharm. 14 (1805) (Lpz.), 47-93. Zit. nach Garrison-Morton, Nr. 1839.

${ }^{3}$ Vgl. J.Landmann, Diagnostik in der ärztlichen Praxis, nach Samuel Gottlieb Vogel (1750-1837), in: Gesnerus 28 (1971), S. 168-195.

${ }^{4}$ L.v. Rechenberg, Die Opiumtherapie in Hufelands Enchiridion medicum (1836), Diss. med., Zürich 1983, Masch.-Schr., vervielfältigt, IV u. $33 \mathrm{~S}$.

5 Nach H.Sallander (Herausg.), Bibliotheca Walleriana, Bd. 1, Stockholm 1955, S. $224 \mathrm{f}$. 
${ }^{6}$ J.Petersen, Hauptmomente in der älteren Geschichte der Medicinischen Klinik, Kopenhagen 1890, S. 260 .

${ }^{7}$ C. Müller, Jeremias Gotthelf und die Ärzte, Bern 1959, S. $34 \mathrm{ff}$.

${ }^{8}$ E. Haffter (Herausg.), Dr.L.Sonderegger in seiner Selbstbiographie und seinen Briefen, Frauenfeld 1898, S. 99.

${ }^{9}$ C. Sachaile de la Barre, Les médecins de Paris jugés par leurs œuvres, ou statistique scientifique et morale des médecins de Paris, Paris 1845, S.551-553.

${ }^{10}$ Universal-Lexicon der pract. Medicin und Chir., Bd. 14, Leipzig 1848, S. 764: Nachwort des Herausgebers. Nach dem Biogr. Lexikon (Hirsch) wanderte d'Alnoncourt nach Konstantinopel aus und starb Mitte der 1850er Jahre in Belgrad.

${ }^{11}$ Wir zitieren Hufelands Enchiridion (vgl. Anm.1) nach der zweiten, vermehrten Auflage von 1836.

12 Dictionnaire de médecine, 2. Aufl., Bd.22, Paris 1830, S.284.

${ }^{13}$ Vgl. auch H.M. Koelbing, Der Urin im medizinischen Denken, Basel 1967 (Documenta Geigy), Heft 6, «Die Harnschau - Lieblingsmethode der Praktiker», S. 85-91.

${ }^{14}$ Das ungebräuchliche und in der lateinischen Sprache nicht vorkommende Wort Exutans für Zugmittel entspricht dem französischen exutoire, vom lat. Verb exuere (exuo, p.p. exatus) herausziehen. Es handelt sich um Mittel, die eine lokale Eiterung bewirken und unterhalten, im Grund also um antike Humoralmedizin.

${ }^{15}$ Vgl. H. M. Koelbing, Medizinische Gesichtspunkte zum Problem der Drogenabhängigkeit, in: Bull. Schweiz. Akad. med. Wissenschaften 27 (1971), S. 58-66.

${ }^{16}$ Nach John Brown (1735-1788) ist Leben gleich Erregung, excitement; Krankheit ist ein Zustand zu großer oder zu geringer Erregung. Je nachdem muß die Therapie dämpfend, sedierend oder aber erregend, stimulierend, tonisierend sein. Brown betrachtete Opium als Tonicum par excellence. - Vgl. H.M. Koelbing, Die ärztliche Therapie, Grundzüge ihrer Geschichte, Darmstadt 1985, Kap.5-6, «Tonisieren und Sedieren als therapeutisches System».

${ }^{17}$ Die Medizinalgewichte verschiedener Staaten stimmten nicht genau überein. Im deutschen Sprachgebiet galt allgemein das alte Nürnberger Medizinalgewicht: 1 Unze (appr. 29,8 g) $=8$ Drachmen $(3,7 \mathrm{~g})=24$ Skrupel $(1,24 \mathrm{~g})=480 \mathrm{Gran}(60 \mathrm{mg})$. Die französischen Werte waren nach Dictionaire des sciences médicales, Bd.1, Paris 1812, «Introduction»: 1 once $(30,6 \mathrm{~g})=8$ gros (oder dragmes, $3,8 \mathrm{~g})=24$ scrupules $(1,27 \mathrm{~g})=576$ grains $(53 \mathrm{mg})$.

${ }^{18}$ Vgl. C. A. Wunderlich, Wien und Paris - ein Beitrag zur Geschichte und Beurteilung der gegenwärtigen Heilkunde in Deutschland und Frankreich, Stuttgart 1841, neue Ausgabe Bern etc. 1974 (Hubers Klassiker der Medizin und der Naturwissenschaften, Bd. 13), S.52: Kritik von Hufelands Entzündungsbegriff; ders., Geschichte der Medicin, Stuttgart 1859, S. $292 \mathrm{f}$.

${ }^{19}$ Vgl. H. M. Koelbing, op. cit. (in Anm. 16), Kap. 5.4, «Quecksilber als Heilmittel».

${ }^{20}$ Vgl. H. M. Koelbing, op. cit. (in Anm.15). - Nach G.Sonnedecker, Die Opiumsucht: Wandlung des Begriffs in historischer Sicht, in: Pharmazeut. Zeitg. 108 (1963), S. 836-840, 899-903, taucht der Ausdruck addiction für die Opiumabhängigkeit bei englischen Autoren schon im 18. Jahrhundert auf. 


\section{Summary}

\section{Hufeland's opium therapy compared with contemporaneous teaching}

In his last work, "Enchiridion medicum" (i.e. "Doctor's Manual"), Christoph Wilhelm Hufeland (1762-1836) praises opium as one of the three foremost means of therapeutics, the other two being bleeding and emetics. Through ten editions (10th ed. 1857) and several translations, the "Enchiridion", first published in 1836, had a considerable influence on practitioners, especially in German speaking countries, down to the middle of the 19th century. A closer examination of Hufeland's recommendations shows, however, a disturbing lack of understanding for the chemical analysis of opium and, therefore, for the importance of morphine as its main component. Clinging to outdated vitalistic concepts, Hufeland ascribes to opium as a whole a depressing effect on the nervous system in combination with a stimulating action on circulation, "the life of blood". This leads him to a wide range of therapeutic indications without exact dosage. A comparison with four medical encyclopaedias published between 1819 and 1841 (two French ones and two German adaptations of French texts), shows that Hufeland and his followers were, already in 1836, lagging behind the science of their time.

On the other hand, Hufeland coined the term of "Opiumsucht" for opium addiction. In very humane words, he recommends opium in order to relieve the pains and distress of terminal illness.

Kontaktadresse:

Prof. Dr. med. Huldrych M. Koelbing

Medizinhistorisches Institut der Universität

Rämistraße 71

CH-8006 Zürich 